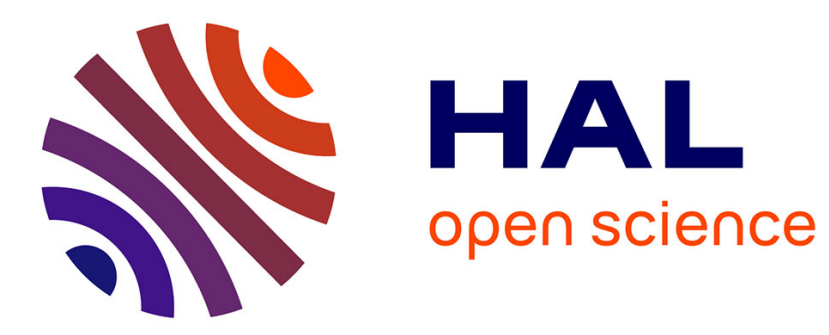

\title{
Interaction of a moving 101-2 twin boundary with perfect dislocations and loops in an HCP metal
}

\author{
Anna Serra, David John Bacon
}

\section{To cite this version:}

Anna Serra, David John Bacon. Interaction of a moving 101-2 twin boundary with perfect dislocations and loops in an HCP metal. Philosophical Magazine, 2010, 90 (07-08), pp.845-861. 10.1080/14786430903023901 . hal-00581019

\section{HAL Id: hal-00581019 \\ https://hal.science/hal-00581019}

Submitted on 30 Mar 2011

HAL is a multi-disciplinary open access archive for the deposit and dissemination of scientific research documents, whether they are published or not. The documents may come from teaching and research institutions in France or abroad, or from public or private research centers.
L'archive ouverte pluridisciplinaire HAL, est destinée au dépôt et à la diffusion de documents scientifiques de niveau recherche, publiés ou non, émanant des établissements d'enseignement et de recherche français ou étrangers, des laboratoires publics ou privés. 


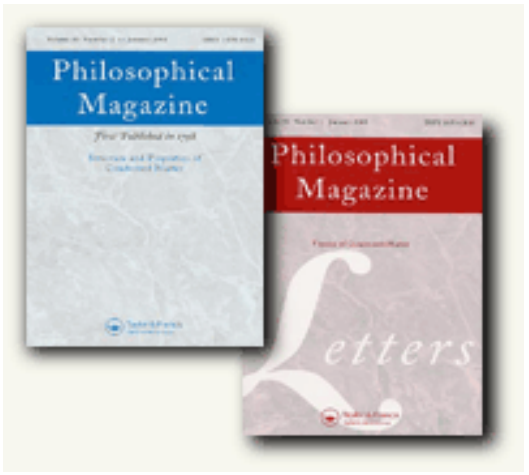

\section{Interaction of a moving $\{101-2\}$ twin boundary with perfect dislocations and loops in an HCP metal}

\begin{tabular}{|r|l|}
\hline Journal: & Philosophical Magazine \& Philosophical Magazine Letters \\
\hline Manuscript ID: & TPHM-09-Mar-0115.R1 \\
\hline Journal Selection: & Philosophical Magazine \\
\hline Author: & 05-May-2009 \\
\hline Complete List of Authors: & $\begin{array}{l}\text { Serra, Anna; Universitat Politècnica de Catalunya (UPC), } \\
\text { Matematica Aplicada III } \\
\text { Bacon, David; The University of Liverpool, Department of } \\
\text { Engineering }\end{array}$ \\
\hline Keywords: & $\begin{array}{l}\text { atomistic simulation, crystal defects, crystalline interface, } \\
\text { deformation twinning, dislocation interactions, topological theory of } \\
\text { defects, twinning }\end{array}$ \\
\hline Keywords (user supplied): & atomistic simulation, crystal defects, crystalline interface \\
\hline & \multicolumn{2}{|l}{} \\
\hline
\end{tabular}

\section{S) ScholaroNE \\ Manuscript Central}


Interaction of a moving $\{10 \overline{1} 2\}$ twin boundary with perfect dislocations and loops in an HCP metal

\author{
A. SERRA ${ }^{1}$ and D.J. BACON ${ }^{2}$ \\ ${ }^{1}$ Dept. Matemàtica Aplicada III, ETSECCPB and CRNE, \\ Universitat Politècnica de Catalunya (UPC), Jordi Girona 1-3, 08034 Barcelona, Spain \\ ${ }^{2}$ Department of Engineering, University of Liverpool, Brownlow Hill, \\ Liverpool L69 3GH, UK
}

\begin{abstract}
Atomic-scale computer simulation is used to investigate the interaction of a moving $[1 \mathbf{1 0}]$ twin boundary in an HCP metal with either a straight $1 / 3<1120>(0001)$ dislocation lying perpendicular to the direction of twinning shear or a periodic row of perfect dislocation loops. The screw dislocation does not decompose in the moving interface and has no effect on its motion. The $60^{\circ}$ mixed dislocation is attracted by the boundary and decomposes into twinning dislocations and a disconnection (an interfacial defect with both step and dislocation character): the sign of the crystal dislocation determines the form of the disconnection and thus its effect on twin boundary motion. Boundary reactions with crystal dislocations are likely to be important for assisting the twinning process. Loops with Burgers vector, $\boldsymbol{b}$, parallel to the interface are reformed in the other crystal after the twin boundary has passed through. The boundary attracts both interstitial and vacancy dislocation loops with inclined $\boldsymbol{b}$ but is not transparent to them, for the complete loop is swept along its glide prism by the moving interface. Depending on its nature, a loop either retains its structure in its parent crystal or is absorbed in the interface. The decomposition product in the latter case is consistent with the reactions of straight dislocations. The results indicate that twinning is efficient at sweeping loops from the microstructure when their density is low and is suppressed by loops when their density is high.
\end{abstract}




\section{Introduction}

Slip in the HCP metals is dominated by dislocations with Burgers vector $\boldsymbol{b}=1 / 3(\mathbf{1 1} \overline{\mathbf{2}} \mathbf{0})$ that glide on either the (0001) basal plane or $\{\mathbf{1 1 0 0}\}$ first-order prism planes. However, the low crystal symmetry limits the number of independent slip systems available for glide of these perfect dislocations and, as a result, plastic deformation by twinning is common. A twin grows into the adjoining crystal by the diffusion-less motion of the interface between them and is therefore influenced, in principle, by crystal defects encountered by the moving interface. This interaction between a twin boundary and other defects is modelled in the present paper by atomic-scale computer simulation. We consider a model for $\alpha$-zirconium $(\mathrm{Zr})$, which is an important metal for nuclear technology, for example. It twins by several different modes, of which $\{1 \mathbf{1 0} \overline{2}\}$ twinning is the most common, i.e. the twin boundary is a $\{10 \overline{12}\}$ plane in both crystals. Such twins are broad due to the high mobility of their boundaries [1].

Earlier computer simulation studies of the interaction between a twin boundary and crystal defects were restricted by the model size and rigid boundary conditions to cases where the twin interface was static and the defects were created sufficiently close to it to react with it spontaneously. This restriction has now been overcome by the development of a boundary condition technique that enables the twin interface to move freely. It allows one crystal in a twin-related pair to expand at the expense of the other by glide of twinning dislocations [2, 3], unrestricted by the boundary conditions (see section 2). The method has already been used to investigate the interaction of a $[10 \overline{12}\}$ twin boundary with single and small clusters of point defects [4], a situation relevant to neutron-irradiated $\mathrm{Zr}$ alloys. However, the defects considered in that paper were small (containing from 2 to 35 point defects) and did not have true dislocation structure.

There are two reasons for broadening the study in the present work to straight dislocations and larger defect clusters. First, the earlier simulations with rigid boundary conditions showed that a straight, perfect dislocation near a $[\mathbf{1 0} \overline{\mathbf{1} 2}]$ twin boundary may be absorbed by it spontaneously, thereby creating an interfacial step with dislocation character [5]. (Such an interfacial line defect is known as a 'disconnection' [6].) Under the application of a suitable shear strain, the disconnection may be a source for creation 
Before proceeding to the model, a brief introduction to the crystallography of the defects studied is required. The admissible interfacial disconnections separating regions of identical boundary structure are best identified using the dichromatic pattern

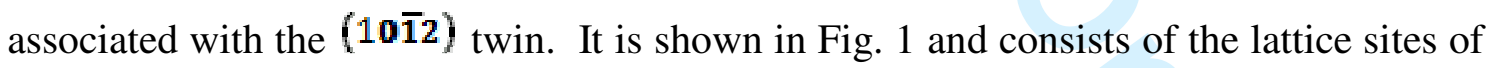
two crystals in twin-related orientation, projected with the sites of a $(\mathbf{1 0 \overline { 1 } 2})$ lattice plane of one crystal coincident with those of a $(\mathbf{1 0 \overline { 1 2 }})$ plane of the other. The two crystals are drawn as white $(\lambda)$ and black $(\mu)$ in Fig. 1 . In addition to the crystal coordinate system it is convenient to define an interfacial coordinate system with $\mathrm{x}$ parallel to the common tilt axis $[\mathbf{1 2} 10]_{\lambda} ; z$ parallel to the normal to the interface and y parallel to the twinning

\footnotetext{
* Twinning dislocations are also disconnections, since they are interfacial steps with dislocation character. Use of the word dislocation for these defects is common, however, and so in this paper we use the terms 'twinning dislocation' and 'twinning disconnection' without distinction.
} 
shear direction [10 $\overline{11}]$. The set of Burgers vectors of admissible interface disconnections is obtained from the difference in the same reference frame between translation vectors $t(\lambda)$ and $t(\mu)$ of the two lattices, defined in the following sense:

$$
b=t(\lambda)-P t(\mu)
$$

where $P$ is the transformation matrix relating the coordinate frames of the two crystals. The sign conventions used here are chosen so that the positive line sense of the disconnection is taken to be out of the paper (+x direction) and $\boldsymbol{b}$ is given by the RH/FS rule, e.g. [10]. Furthermore, the sense of step 'up' or 'down' refers to a boundary being traversed from left to right, i.e. in the $+\mathrm{y}$ direction. Burgers vectors are written as $\boldsymbol{b}_{ \pm \mathrm{m} / \pm \mathrm{n}}$, where the magnitude of the subscripts $m$ and $n$ indicates the number of (10i2) lattice planes, spacing $d$, at the step in the upper and lower crystals, respectively, and the subscript sign indicates the step sense. Thus, a twinning dislocation has Burgers vector $\boldsymbol{b}_{2 / 2}$ (step up) or $\boldsymbol{b}_{-2 /-2}$ (step down). The latter is shown in Fig. 1 and is the difference between $\boldsymbol{t}_{\lambda}=[\overline{\mathbf{1 0 1 0}}]_{\lambda}$ defining a step down by two planes on the lower surface of the $\lambda$ crystal, and $\boldsymbol{t}_{\mu}=[0001]_{\mu}$ defining a step down by two planes on the upper surface of the $\mu$ crystal. The same notation is adopted for crystal dislocations, i.e. $\boldsymbol{b}$ of a dislocation of the type $1 / 3(11 \overline{2} 0\rangle$ at $60^{\circ}$ to the $\mathrm{x}$ axis in the $\lambda$ crystal is labelled $\boldsymbol{b}_{1 / 0}$ in Fig. 1 and is defined by $\boldsymbol{t}_{\lambda}$ that steps up by one $(\mathbf{1 0} \overline{\mathbf{1}})$ lattice plane in $\lambda$, whereas $\boldsymbol{b}_{-1 / 0}$ corresponds to $\boldsymbol{t}_{\lambda}$ that steps down by one plane. (With the two-subscript notation for Burgers vectors, the magnitude and sign of each subscript is conserved in dislocation and disconnection reactions, as will be seen in section 3 .)

\section{Model}

The computational model had about one million atoms (dimensions $L_{\mathrm{x}}, L_{\mathrm{y}}, L_{\mathrm{z}}=23.4$, $35.5,28.9 \mathrm{~nm}$ ) and consisted of two half-crystals in twin orientation separated by either a planar twin boundary or one containing a twinning disconnection in edge orientation with line direction parallel to the $\mathrm{X}$-axis $[\mathbf{1 2} 10]_{\lambda}$ and Burgers vector $\boldsymbol{b}_{-2 / 2}=\alpha[\mathbf{1 0} \overline{\mathbf{1 1}}]: \alpha$ is approximately 0.0645 for the lattice parameter ratio $c / a=1.5952$ (see [1] for details). This bi-crystal model had periodic boundary conditions in the interfacial glide plane $x-y$ of the twinning disconnection and fixed outer boundaries in the form of rigid blocks of 
atoms in the $\pm \mathrm{z}$ directions $[2,3]$. With this construction, when a twinning disconnection moving in the $\pm y$ direction crosses one periodic boundary, it re-enters at the opposite side of the simulation box and, due to the step character of the disconnection, the twin interface is displaced in the $\mathrm{z}$ direction by the step height $\pm 2 d$. Crystal dislocations or dislocation loops were introduced on either side of the twin boundary. To assist understanding of the reactions and mechanisms found, atoms with irregular coordination number were recorded, as will be shown in the figures that follow. Most of the modelling used molecular statics simulations, i.e. temperature $T=0 \mathrm{~K}$. In these, the $\boldsymbol{b}_{-2 /-2}$ disconnection was induced to glide by applying shear strain $\mathrm{e}_{\mathrm{yz}}$ in increments of $10^{-5}$ via displacement of the upper rigid block relative to the lower one. (The $\boldsymbol{b}_{-2 /-2}$ defect has its extra half-plane in the upper $(\lambda)$ crystal and glides to the right $(+y)$ under positive applied shear strain.) The atomic system was relaxed to minimise the potential energy after each shear displacement using the interatomic potential for $\mathrm{Zr}$ from [11] that we refer to as A95. The applied resolved shear stress for each level of strain was calculated from the resultant force on the upper rigid block, as described in [12]. To check the stability of some configurations, molecular dynamic simulations for $T=100 \mathrm{~K}$ were performed using two different interatomic potentials, namely A95 and the potential described in [13], which we denote as M07. In these cases, strain was applied at a constant rate of $25 \times 10^{6} \mathrm{~s}^{-1}$ by applying a constant velocity to the upper rigid block of atoms. The principal difference between the two potentials arises from the value of the intrinsic stacking fault energy on the basal and first-order prism planes. The fault energy is relatively low on the basal plane in the A95 model, resulting in a preference for dissociation into two Shockley partials. Dissociation also occurs in the M07 model, but the partial spacing is much smaller [14].

\section{Interaction of a straight dislocation with the $(10 \overline{1} 2)$ twin boundary}

\subsection{Dislocations considered}

A straight perfect dislocation with line direction along the $\mathrm{x}$ axis $[\mathbf{1 2} 10]_{\lambda}$ can be pure screw, i.e. $\boldsymbol{b}=1 / 3[\mathbf{1 2} 10]_{\lambda}$, or have $60^{\circ}$-mixed character, i.e. $\boldsymbol{b}= \pm 1 / 3[1 \mathbf{1 1 2} 0]_{\lambda}$ or $\boldsymbol{b}=$ $\pm 1 / 3[\overline{\mathbf{2}} 110]_{\lambda}$. The latter vectors are represented by $\boldsymbol{b}_{ \pm 1 / 0}$ in the dichromatic pattern of Fig. 1. However, only the $60^{\circ}$ cases are considered here, for two reasons. First, it was 
shown in [15] that the screw dislocation gliding under stress in the $\lambda$ crystal is either transmitted through the twin boundary into the $\mu$ crystal or is reflected back into the $\lambda$ crystal with a change of glide plane, e.g. basal-to-prism or vice versa. Decomposition into products that change the structure of the boundary does not occur. Second, it was found in [5] that a $60^{\circ}$-mixed dislocation decomposes when entering the interface, thereby changing its structure and creating interfacial disconnections. More detail follows below. Note that perfect dislocation loops have the same $1 / 3\langle 11 \overline{2} 0\rangle_{\lambda}$ Burgers vectors, and for these defects it is necessary to consider all three orientations of $\boldsymbol{b}$, as will be seen in section 4 .

The interaction between the twin boundary and a $60^{\circ}$ crystal dislocation gliding on the basal plane depends on the sign of the dislocation and the order of the two Shockley partials relative to the boundary. (If the basal glide plane of the crystal dislocation lies between planes $\mathrm{A}$ and $\mathrm{B}$, say, in the ... $\mathrm{ABAB} \ldots$ stacking sequence, the order of the Shockley partials is reversed from that when the glide plane lies between B and A.) The partials have Burgers vectors $\boldsymbol{b}^{30}$ and $\boldsymbol{b}^{90}$ at $30^{\circ}$ and $90^{\circ}$ to the line direction, respectively. The interaction of $60^{\circ}$ dislocations of opposite line sense with the twin interface was reported in [16] for a model $\alpha$-Ti simulated in a box containing about

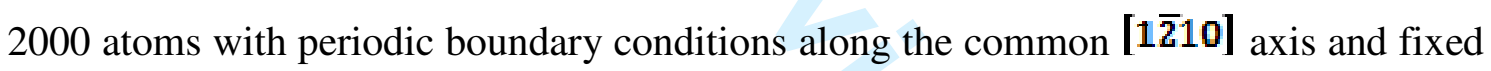
boundary conditions in the other directions. These early results illustrated the reaction at the boundary but it was not possible to study the mobility of the resulting interfacial line defects nor the interaction of such defects with pre-existing twinning dislocations. In the present study we revisit the previous results using the much bigger simulation box with incorporation of the new periodic boundary conditions in the glide plane, i.e. the twinning plane [2, 3], that permit movement of twinning dislocations over large distances in the model. This allows twinning dislocations to interact repeatedly with the interfacial defect(s) formed by decomposition of a crystal dislocation.

Unlike the previous work where a single dislocation parallel to the $\mathrm{x}$ axis was introduced in the $\lambda$ crystal, it was necessary to create a dipole pair of straight dislocations of opposite sign with $\boldsymbol{b}=1 / 3(\mathbf{1 1} \overline{\mathbf{2}} \mathbf{0})$ in order to retain the periodicity of the simulation box. One of the pair was much closer than the other to the interface and so interacted with it alone and decomposed as a single dislocation. When motion of the 
boundary occurred by glide of one or more twinning disconnections under continued stress, it was then able to move closer to the second, more distant dislocation of the dipole and another reaction could occur, but this time with a dislocation of opposite sign to the first.

Fig. 2 is a schematic summary to be discussed below of the results of the earlier simulations for the model with a small number of atoms and fixed boundary conditions [16]. There are three different possibilities for the reaction, depending on the sign of the dislocation line and the stacking sequence of the basal plane on which it glides. In the Burgers vector notation used, $\boldsymbol{b}$ of the reacting crystal dislocation is either $\boldsymbol{b}_{1 / 0}$ or $\boldsymbol{b}_{-1 / 0}$, as indicated. The outcomes are as follows.

\subsection{Dislocation with $\boldsymbol{b}=\boldsymbol{b}_{1 / 0}$}

The dichromatic pattern in Fig. 1 shows a set of possible reactions represented by the following Burgers vector balance:

$$
\boldsymbol{b}_{1 / 0}=\boldsymbol{b}_{-(2 \mathrm{n}-1) /-2 \mathrm{n}}+n \boldsymbol{b}_{2 / 2}
$$

The magnitude of $\boldsymbol{b}_{-(2 \mathrm{n}-1) /-2 \mathrm{n}}$ depends on the height of the step $(n d)$ and the c/a ratio: when c/a just less than the ideal value $(8 / 3)^{1 / 2}$, as in $\mathrm{Zr}$, the smallest magnitudes correspond to $n=3$ and 4 (see Fig. 1). The dislocation is attracted to, and absorbed spontaneously by, the twin boundary in this reaction when less than about $8 \mathrm{~nm}$ from it. In [5] it decomposed into a $\boldsymbol{b}_{-5 /-6}$ disconnection line and three $\boldsymbol{b}_{2 / 2}$ twinning disconnections (Fig. 2(a)). In the larger, periodic model of the present work, it formed a $\boldsymbol{b}_{-7 /-8}$ defect and four $\boldsymbol{b}_{2 / 2}$ defects. This outcome was found to be independent of the leading/trailing order of the partials of the crystal dislocation. If the boundary initially contains a $\boldsymbol{b}_{-2 /-2}$ disconnection, it annihilates with one of the $\boldsymbol{b}_{2 / 2}$ defects, leaving only three. The periodicity of the simulated box allows each $\boldsymbol{b}_{2 / 2}$ twinning dislocation to move freely under the influence of the others and they spread equidistantly along the interface.

Under applied shear strain $\mathrm{e}_{\mathrm{yz}}>0$, the $\boldsymbol{b}_{2 / 2}$ disconnections glide to the left (-y direction). The critical resolved shear stress, $\tau_{\mathrm{c}}$, necessary for the upper block to move a distance $\left|\boldsymbol{b}_{2 / 2}\right|$, i.e. for the total distance moved by the four (or three) defects to equal $L_{\mathrm{y}}$, is 
27MPa. This value represents the obstacle stress when a $\boldsymbol{b}_{2 / 2}$ twinning dislocation encounters a $\boldsymbol{b}_{-7 /-8}$ disconnection and overcomes it by conservative climb and subsequent detachment on the other side. (It was shown in [16] that climb of a $\boldsymbol{b}_{ \pm 2 / \pm 2}$ defect from one side of $\boldsymbol{a} \boldsymbol{b}_{-7 /-8}$ disconnection to the other is compensated by climb of the latter along its riser plane as the twin boundary translates by $\pm 2 d$.) Furthermore, it has been shown that if the $\boldsymbol{b}_{-7 /-8}$ disconnection exists in the interface in the absence of pre-existing twinning dislocations, it can act as a source of dipoles containing pairs of equal and opposite $\boldsymbol{b}_{ \pm 2 / \pm 2}$ defects when $\tau$ reaches a value of $110 \mathrm{MPa}[14]$.

Thus, the disconnections such as $\boldsymbol{b}_{ \pm 7 / \pm 8}$ and $\boldsymbol{b}_{ \pm 5 / \pm 6}$ can be created by the decomposition of crystal dislocations at the twin boundary and are effective sources of $\boldsymbol{b}_{ \pm 2 / \pm 2}$ twinning dislocations. They move with the twin boundary by conservative climb along their riser $[5,16]$, thereby supplying the twinning disconnections necessary to keep the boundary moving.

\subsection{Dislocation with $\boldsymbol{b}=\boldsymbol{b}-1 / 0$}

The reaction is more complex since although three disconnections form in the boundary, one with $\boldsymbol{b}_{4 / 4}$ and two with $\boldsymbol{b}_{-2 /-2}$, the $\boldsymbol{b}_{-1 / 0}$ dislocation is not fully absorbed. Instead, only the leading partial of the $\boldsymbol{b}_{-1 / 0}$ line is attached to the $\boldsymbol{b}_{4 / 4}$ disconnection. The trailing partial stands off from the boundary, separated from it by an intrinsic basal stacking fault. The leading Shockley partial stabilizes the $\boldsymbol{b}_{4 / 4}$ disconnection because this defect on its own is not stable and decomposes into two $\boldsymbol{b}_{2 / 2}$ twinning dislocations, in accordance with Frank's rule. We can therefore describe the reaction as

$$
\boldsymbol{b}_{-1 / 0}=\left(\boldsymbol{b}_{4 / 4}+\boldsymbol{b}^{\mathrm{L}}\right)+\boldsymbol{b}^{\mathrm{T}}+2 \boldsymbol{b}_{-2 /-2}
$$

where $\boldsymbol{b}^{\mathrm{L}}$ and $\boldsymbol{b}^{\mathrm{T}}$ are the Burgers vectors of the leading and trailing partials respectively. The outcome is represented schematically in Fig. 2(b).

The reaction of eq. (3) and Fig. 2(b) is the same as that found with the smaller atomic model in [16] when the leading partial has $\boldsymbol{b}^{\mathrm{L}}=\boldsymbol{b}^{90}$. However, it was reported in [16] that when the leading partial has $\boldsymbol{b}^{\mathrm{L}}=\boldsymbol{b}^{30}$ the following reaction occurs: 


$$
\boldsymbol{b}_{-1 / 0}=\boldsymbol{b}_{3 / 4}+2 \boldsymbol{b}_{-2 /-2}
$$

as sketched in Fig. 2(c). The present work shows that this configuration is not the most favourable if the number of mobile atoms in the simulation box is big enough and the disconnections can move freely along the interface under their mutual interaction. Moreover, the complex consisting of a $\boldsymbol{b}_{4 / 4}$ disconnection linked to a $\boldsymbol{b}_{-1 / 0}$ crystal dislocation is more suitable for conservative motion of the facetted boundary under the influence of gliding twinning dislocations, as described at the end of this sub-section.

Under a shear strain $\mathrm{e}_{\mathrm{yz}}>0$, glide of twinning dislocations on the interface has two possible outcomes. A $\boldsymbol{b}_{2 / 2}$ dislocation approaches the $\left(\boldsymbol{b}_{4 / 4}+\boldsymbol{b}^{\mathrm{L}}\right)$ complex from the right and initially converts $\boldsymbol{b}_{4 / 4}$ to $\boldsymbol{b}_{6 / 6}$, but this disconnection is unstable and emits another $\boldsymbol{b}_{2 / 2}$ twinning dislocation that glides to the left at a resolved stress of 70MPa. When a $\boldsymbol{b}_{-2 /-2}$ dislocation approaches from the left, on the other hand, the $\boldsymbol{b}_{4 / 4}$ defect is converted to $\boldsymbol{b}_{2 / 2}$ plus a $\left(\boldsymbol{b}_{2 / 2}+\boldsymbol{b}_{-2 /-2}\right)$ dipole. The $\boldsymbol{b}_{-2 /-2}$ dislocation eventually glides away to the right when the applied shear stress reaches 80MPa. In both cases, the $\left(\boldsymbol{b}_{4 / 4}+\boldsymbol{b}^{\mathrm{L}}\right)$ complex is restored but in a boundary that is displaced by $2 d$ in the $z$ direction.

As the positive shear strain is increased further, the second crystal dislocation of the dipole pair in the upper crystal is absorbed by the boundary. This $\boldsymbol{b}_{1 / 0}$ dislocation is totally absorbed, thereby creating a $\boldsymbol{b}_{-5 /-6}$ disconnection and three $\boldsymbol{b}_{2 / 2}$ twinning dislocations, as in Fig. 2(a). Two of the latter are annihilated with the two $\boldsymbol{b}_{-2 /-2}$ defects associated with the $\boldsymbol{b}_{-1 / 0}$ component of the dipole. The resulting structure is shown by the visualization of the interface and defect core atoms in Fig. 3. The projection direction is near $[\mathbf{1 2} 10]_{\lambda}$ and the initial and final defects are indicated by the dislocation symbols. The resolved shear stress to move the remaining $\boldsymbol{b}_{2 / 2}$ defect along the boundary containing the $\boldsymbol{b}_{4 / 4}$ and $\boldsymbol{b}_{-5 /-6}$ disconnections is $80 \mathrm{MPa}$, the controlling obstacle being the $\boldsymbol{b}_{4 / 4}$ riser as explained above.

The direction of movement of the $\boldsymbol{b}_{-2 /-2}$ twinning dislocations is reversed when a negative shear strain $\left(\mathrm{e}_{\mathrm{yz}}<0\right)$ is applied and the interface containing the $\left(\boldsymbol{b}_{4 / 4}+\boldsymbol{b}^{\mathrm{L}}\right)$ configuration formed by reaction (3) moves downwards by their repeated glide. Their interaction mechanism with the complex is equivalent to that just described. The other crystal dislocation with $\boldsymbol{b}=\boldsymbol{b}_{1 / 0}$ in the upper crystal does not enter the boundary in this 
case. It is interesting to note that the $\boldsymbol{b}_{4 / 4}$ disconnection resulting from the partial absorption of the $\boldsymbol{b}_{-1 / 0}$ crystal dislocation by reaction (3) has a riser lying almost perpendicular to the basal planes of the upper $(\lambda)$ crystal. However, when it moves as the boundary is translated by $\pm 2 d$ every time a $\boldsymbol{b}_{ \pm 2 / \pm 2}$ twinning dislocation glides across, it is displaced along a basal plane of the $\lambda$ crystal. This motion of the $\boldsymbol{b}_{4 / 4}$ defect perpendicular to its own riser facet is conservative, i.e. climb is not involved. It occurs because it takes place along the glide plane of the $\boldsymbol{b}_{-1 / 0}$ crystal dislocation and the core of this dislocation is retained in association with the riser.

\section{Interaction of a perfect dislocation loop with the twin boundary}

\subsection{Loop geometry}

It has been shown by computer simulation [17] that in their most stable form, small perfect dislocation loops with $\boldsymbol{b}=1 / 3\langle\mathbf{1 1} \overline{\mathbf{2}} \mathbf{0}\rangle$ are rectangular in shape and have pure edge orientation with habit plane $[1 \mathbf{1 2} 0]$. Two of the sides lie in the [0001] direction and are not dissociated; the others lie in a $\langle\mathbf{1 0 \overline { \mathbf { 1 } } \mathbf { 0 }}\rangle$ direction in the basal plane and are dissociated, with partial spacing dependent on the stacking fault energy given by the interatomic potential. Interstitial and vacancy loops with approximate dimensions $5 \times 9 \mathrm{~nm}$ containing 627 point defects were simulated in the present work.

Loops in the three possible $[1 \overline{120}]$ planes have been considered here. As with the straight dislocation in section 3 , the Burgers vector of such a loop is either parallel to the common [1 $\overline{2} 10] \mathrm{x}$-axis of the two half-crystals or at an angle of $60^{\circ}$ to this axis. A loop with $\boldsymbol{b}=1 / 3[\mathbf{1} \overline{\mathbf{2}} 10]$ cannot approach the twin boundary by glide and therefore interaction only occurs due to the movement of the twin boundary towards the cluster itself, i.e. by glide of pre-existing $\boldsymbol{b}_{ \pm 2 / \pm 2}$ defects in the twin boundary under applied stress. We treat this case first in section 4.2. A $60^{\circ}$ loop with Burgers vector $\boldsymbol{b}_{ \pm 1 / 0}$ can react with the twin boundary either in the same way, i.e. by motion of the twin boundary, or by glide on its glide prism due to attraction by the boundary. Unlike the $60^{\circ}$ dislocation in section 3 , which has a line direction along the $\mathrm{x}$-axis parallel to the twin boundary, a $60^{\circ}$ loop meets the boundary first at one of its corners. The two $60^{\circ}$ - 
loop orientations are equivalent by symmetry, so only one need be considered. Results for this case are described in section 4.3.

\subsection{Loops with $\boldsymbol{b}$ parallel to the boundary}

(a) Interstitial loops

Since a loop in this orientation cannot be dragged when the boundary moves, it offers strong resistance to the glide of a twinning dislocation. For the boundary to overcome this obstacle, the twinning dislocation has to interact with the loop on successive cycles across the model. During this process the loop can move only along the twin boundary by a thermally activated motion. The repeated interaction of the loop with twinning dislocations reorients it from one crystal to the other, i.e. the sides lying in the basal and prism planes in the $\lambda$ crystal become sides in the prism and basal planes of the $\mu$ crystal, respectively. (The basal and prism planes in $\lambda$ make an angle of $4.7^{\circ}$ with the prism and basal planes in $\mu$ (see Fig. 1).)

Fig. 4 shows visualisations of the loop and boundary containing a $\boldsymbol{b}_{-2 /-2}$ disconnection at different stages of the process. Fig. 4(a) is a near [12 10$]$ projection as the $\boldsymbol{b}_{-2 /-2}$ defect approaches the loop from the left and the twin boundary is about to meet the lower corner of the loop. The conversion of the loop from $\lambda$ to $\mu$ is half-complete in Fig. 4(b), where the $\mathrm{x}$-axis projection shows clearly the transformation of the loop sides from basal to prism and vice versa. The loop acts as an obstacle to the repeated glide of the $\boldsymbol{b}$. 2/-2 twinning disconnection, as can be seen in Fig. 4(c) by the $\mathrm{z}$-axis projection of the configuration shown in (b). The $\boldsymbol{b}_{-2 /-2}$ dislocation is seen to be bent strongly as it breaks away from the loop. The critical stress for this is $60 \mathrm{MPa}$.

If the centre of the original loop in the $\lambda$ crystal is shifted by $1 / 2$ [0001], so that the row of interstitials at the lower basal-plane side is changed from, say, an A layer to a B layer in the ...ABAB... stacking sequence of (0001) planes, the leading-trailing order of the basal-plane Shockley partials is reversed and an alternative process is observed when the moving boundary first meets the loop corner. It is shown in Fig. 4(d), in which a loop of $\boldsymbol{b}_{-2 /-2}$ twinning disconnection has been nucleated in the twin boundary at the loop corner. (The dissociated nature of the two sides in basal planes is clear in this view: the stacking fault between the partials is not visualised). This nucleation occurs with 
every circuit of the pre-existing $\boldsymbol{b}_{-2 /-2}$ dislocation. The left-hand side of the loop attracts the incoming $\boldsymbol{b}_{-2 /-2}$ defect and assists its passage through the loop. The critical shape is similar in appearance to that in Fig. $4(\mathrm{c})$, but $\tau_{\mathrm{c}}$ is reduced to $40 \mathrm{MPa}$.

The difference in the mechanisms described in the previous two paragraphs is believed to arise from the atomic structure of the $[10 \overline{12}]$ twin boundary. As discussed in [15],

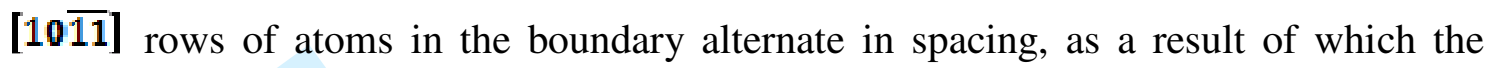
hydrostatic stress alternates between positive and negative along a row. Consequently, the atomic configuration of the interstitial atom at the lowest corner of a loop is not the same for the two situations described above. However, in neither case are any point defects removed from or added to the loop and it is simply transformed from the $\lambda$ crystal to the $\mu$ crystal with the same shape, size and Burgers vector.

(b) Vacancy loops

Interaction between the twin boundary and a vacancy loop with $\boldsymbol{b}$ parallel to the x-axis of the model results in the generation of defects in the $\mu$ crystal, unlike the situation described for the interstitial loop. This is demonstrated by the two stages in the process shown in Figs. 5(a) and (b). The loop in the $\mu$ crystal after the boundary has passed through is seen to be accompanied by other defects, which are vacancy clusters. The transformed loop is smaller by about $28 \%$ than the loop created originally in the $\lambda$ crystal. It has not been possible to discern the precise details of the mechanism involved, but it appears that the vacancies start to form and finally become detached from the loop as the $\boldsymbol{b}_{-2 /-2}$ disconnections pass through the loop corners. The process results in an increase in the potential energy of the bicrystal, as can be seen in the plot of change of potential energy versus applied shear strain in Fig. 5(c). Whereas the energy of the system is restored to its original value when the interstitial loop is reformed in the $\mu$ crystal, it is increased permanently by the breakup of the vacancy loop. There is a corresponding increase in the maximum stress to $130 \mathrm{MPa}$, which occurs at the stage when the vacancy clusters are released from the loop in their final form.

\subsection{Loops with $\boldsymbol{b}$ at $60^{\circ}$ to the $\mathrm{x}$ axis}

a) Interstitial loops 
The configuration with the loop initially in the $\lambda$ crystal is visualised in Fig. 6(a). (This is for the M07 potential, which gives a higher energy for the basal stacking fault than the A95 potential, and hence results in small spacing of the partials that form the basalplane sides.) We denote the lower and upper basal-plane sides BS1 and BS2, respectively, as indicated. Unlike a loop with $\boldsymbol{b}$ parallel to $\mathrm{x}$-axis, the $60^{\circ}$ loops do not transform to equivalent loops in the $\mu$ crystal, i.e. the boundary is not transparent to them. Instead the interstitials in these loops are transported in the $\lambda$ crystal by the moving twin boundary. The way in which they are transported has various forms. In the simplest case, the loop does not make contact with the twin boundary but is pushed ahead of it as the $\boldsymbol{b}_{-2 /-2}$ twinning disconnection sweeps repeatedly across the interface plane. This is shown by the visualisation in Fig. 6(a), which is for the M07 model at $T$ $=0 \mathrm{~K}$. This outcome is independent of the AB or BA order in the ...ABAB ... basal-plane stacking of the interstitials in side BS1.

In the model based on the A95 interatomic potential, the loop rotates on its glide prism until side BS1 lies in the twin boundary, where it reacts with it in a manner analogous to the infinite straight dislocation in section 3. With the $30^{\circ}$ partial leading, a step is formed in the interface and two $\boldsymbol{b}_{-2 /-2}$ disconnections are generated, as can be seen in Fig. 6(b). The other three sides of the loop remain separate from the twin boundary. As the boundary moves upwards into the $\lambda$ crystal by glide of the pre-existing $\boldsymbol{b}_{-2 /-2}$ twinning dislocation, the loop moves upwards along its glide prism with side BS1 incorporated in the twin boundary. The repeated emission of the two $\boldsymbol{b}-2 /-2$ defects assists the motion of the twin boundary and the maximum $\tau$ for the process is only $20 \mathrm{MPa}$. When the centre of gravity of the loop is shifted by $1 / 2[0001]_{\lambda}$ so that the $90^{\circ}$ partial leads the entry of BS1 into the twin boundary, all four sides of the loop are absorbed into the twin boundary, as shown in Fig. 6(c). As the twin boundary moves upwards under applied stress, it drags the absorbed interstitial cluster along the $60^{\circ}$ direction of the glide prism of the original loop. This process provides a strong obstacle to the glide of the $\boldsymbol{b}_{-2 /-2}$ twinning dislocation and $\tau_{\mathrm{c}}$ is 55MPa. MD simulations of $T=$ $100 \mathrm{~K}$ showed the same features.

\section{a) Vacancy loops}

The $60^{\circ}$ vacancy loops are absorbed on the twin boundary by glide and rotation on their glide prism, irrespective of the $30^{\circ}$ or $90^{\circ}$ nature of the leading partial of side BS1. This 
is demonstrated clearly by the $\mathrm{x}$-axis projection in Fig. 7(a). The reaction product of the dislocation segments is consistent with that found in section 3 for the corresponding straight dislocation. Thus, the left part due to BS1 forms a $\boldsymbol{b}_{-5 /-6}$ segment in the form of a step down of the boundary inside the loop: this is analogous to the reaction sketched in Fig. 2(a) for the straight $60^{\circ}$ dislocation. The right part due to side BS2 forms a $\boldsymbol{b}_{3 / 4}$ segment, analogous to the product in Fig. 2(b). The absorbed vacancy cluster moves together with the twin boundary by conservative glide along the glide prism of the original loop.

The absorbed loop reacts with the pre-existing $\boldsymbol{b}_{-2 /-2}$ disconnection in an asymmetric way as it glides across the boundary. This is shown in the z-axis projection of Fig. 7(b), where the image from the atomic cell has been duplicated along the periodic direction for clarity. Whereas the $\boldsymbol{b}_{-2 /-2}$ twinning disconnection is pinned in the lower side of the absorbed loop in the Figure, its movement is enhanced in the upper part. (These sides were the prism-plane sides of the original loop.) This enhancement is due to a $\boldsymbol{b}_{2 / 2}$ disconnection loop that is created in the upper part of the defect and joins the interacting twinning dislocation. The obstacle strength of the vacancy loop to the glide of the $\boldsymbol{b}_{-2 /-2}$ twinning dislocation is less than that of the interstitial loop and $\tau_{\mathrm{c}}$ is $40 \mathrm{MPa}$.

\section{Discussion and Conclusions}

The development of a method that allows an interface containing a disconnection line, i.e. a defect with step as well as dislocation character, to be simulated with periodic boundary conditions in the interface plane has allowed us to investigate the interaction of a moving twin boundary with two types of crystal defect, namely a straight, infinitely long dislocation line in a basal plane and a periodic row of perfect dislocation loops. The interface considered is that of the $\{1 \mathbf{1 0} 2\}$ twin, which is important and common in the deformation of the HCP metals. The interaction between straight crystal dislocations of the $1 / 3<1120>(0001)$ slip system and the $\{10 \overline{1}]$ twin interface have been modelled previously, but by simulation where the number of atoms in the model was small and fixed boundary conditions were employed, as a consequence of which the interface was unable to be translated through the model by glide of twinning dislocations. With this restriction overcome, it has been possible to revisit the 
We have considered straight, $1 / 3<1120>(0001)$ crystal dislocations lying parallel to the interface and perpendicular to the direction of twinning shear. The screw dislocation parallel to the interface does not decompose when it meets the moving interface and has no discernible effect on its motion. The $60^{\circ}$ mixed dislocation is attracted by the boundary in all situations, but the form of the decomposition product and its effect on twin boundary motion depend on the sign of the dislocation $\left(\boldsymbol{b}_{ \pm 1 / 0}\right.$ in the notation here). The dislocation with $\boldsymbol{b}=\boldsymbol{b}_{1 / 0}$ has its extra half-plane intersecting the interface and fully decomposes in it to form a $\boldsymbol{b}_{-7 /-8}$ disconnection and four $\boldsymbol{b}_{2 / 2}$ twinning dislocations, independently of the nature of the leading/trailing Shockley partials (Fig. 2(a)). The current simulations confirm the earlier finding [5] that this disconnection moves with the twin boundary along its riser (the basal plane of one of the crystals) because twinning dislocations can move past it by glide and self-compensated climb. Furthermore, this disconnection acts as a source of twinning dislocations when stress is applied in the absence of pre-existing twinning dislocations. Thus, this decomposition product provides a simple mechanism for twin growth/shrinkage, as predicted in [5].

The decomposition product of a $60^{\circ}$ dislocation of opposite sign $\left(\boldsymbol{b}=\boldsymbol{b}_{-1 / 0}\right)$ with its extra half-plane directed away from the interface is more complex (Fig. 2(b)). Three disconnections form in the boundary, one with $\boldsymbol{b}_{4 / 4}$ and two with $\boldsymbol{b}_{-2 /-2}$, but the $\boldsymbol{b}_{-1 / 0}$ dislocation is not fully absorbed. Instead, only the leading partial of the $\boldsymbol{b}_{-1 / 0}$ line is held in the core of the $\boldsymbol{b}_{4 / 4}$ disconnection (Fig. 2(b)). The $\left(\boldsymbol{b}_{4 / 4}+\boldsymbol{b}^{\mathrm{L}}\right)$ complex thus formed is stable under positive and negative shear strain $\mathrm{e}_{\mathrm{yz}}$ and acts as an obstacle to the glide of $\boldsymbol{b}_{ \pm 2 / \pm 2}$ twinning dislocations. The critical resolved shear stress for twinning dislocations to glide over the interface rises to $70-80 \mathrm{MPa}$.

The $\boldsymbol{b}_{4 / 4}$ disconnection resulting from the partial absorption of the $\boldsymbol{b}_{-1 / 0}$ crystal dislocation has a riser lying perpendicular to the basal planes of the upper $(\lambda)$ crystal. 
However, when it moves with the boundary, which is translated by $\pm 2 d$ every time a $\boldsymbol{b}_{ \pm 2 / \pm 2}$ twinning dislocation glides across, it is displaced along a basal plane of the $\lambda$ crystal together with the $\boldsymbol{b}_{-1 / 0}$ crystal dislocation. The motion of the $\boldsymbol{b}_{4 / 4}$ defect perpendicular to its own riser occurs because of the reaction of $\boldsymbol{b}_{4 / 4}$ with $\boldsymbol{b}_{ \pm 2 / \pm 2}$ (resulting in $\boldsymbol{b}_{6 / 6}$ or $\boldsymbol{b}_{2 / 2}$ ) every time a twinning dislocation sweeps over the boundary and, therefore, is conservative, i.e. climb is not involved.

We conclude that $\boldsymbol{b}_{ \pm 1 / 0}$ crystal dislocations are attracted to and trapped by the twin boundary. They can be fully absorbed $\left(\boldsymbol{b}_{-7 / 8}\right.$ defect) or partially attached to a $\boldsymbol{b}_{4 / 4}$ defect, but in both cases they are pulled/pushed conservatively by the twin boundary along a basal-plane.

The perfect $1 / 3<\mathbf{1 1 2 0}>$ dislocation loops considered are rectangular with sides lying in the basal and first-order prism planes. Those with $\boldsymbol{b}$ inclined to the twin interface (at $60^{\circ}$ to the $\mathrm{x}$-axis of the model) are attracted to it and one of several reactions occurs. It has been seen that the twin boundary is not transparent to either vacancy or interstitial loops. Instead, the complete loop is swept along its glide prism by the moving interface. This is a mechanism by which deformation twins can remove radiation damage from an irradiated microstructure. The precise atomic configuration adopted by a loop in this sweeping process depends on its nature, the leading/trailing order of the partials in the basal-plane side that first meets the interface, and the interatomic potential. In two cases, it was seen to retain the loop structure in its parent crystal, but in others it was absorbed in the interface itself. The decomposition of the loop sides in the interface when this takes place can be explained in terms of the reactions found for straight dislocations. Loops with $\boldsymbol{b}$ parallel to the interface are reformed in the other crystal after the twin boundary has passed through, although in the vacancy case it is smaller because some of its vacancies become detached from the loop.

By comparing the applied stress magnitudes for glide of twinning dislocations at $0 \mathrm{~K}$ after reactions with crystal defects have occurred, it is clear that the disconnections formed by both long straight dislocations and loops raise the stress for continuous motion of the twin interface. However, in the case of long dislocations, several twinning dislocations are formed in each reaction and the disconnection itself can act as 


\section{Acknowledgements}

Discussions with Dr. Yu N Osetsky are acknowledged. A.S. was supported by the Spanish Ministry of Science and Innovation (FIS2006-12436-C02-02) and the Catalan Government (CIRIT 2005 SGR - 00779). The computing was carried out in CESCA (www.cesca.es). 


\section{References}

1. A. Serra, R.C. Pond and D.J. Bacon, Acta Metall. Mater., 39, 1469 (1991).

2. A. Serra and D.J. Bacon, Zeitschrift für Metallkunde, 95, 242 (2004).

3. A. Serra and D.J. Bacon, Mat. Sci. Eng. A, 400, 496 (2005).

4. A. Serra, D.J. Bacon and Yu.N. Osetsky, Phil. Mag. Lett., 87, 451 (2007).

5. A. Serra and D.J. Bacon, Phil. Mag., 73, 333 (1996).

6. R.C. Pond: in Dislocations in Solids, F.R.N. Nabarro, ed., North Holland, Amsterdam, 1989, vol. 8, p. 1.

7. M Griffiths, , Phil. Mag. A, 63, 835 (1991).

8. Y. Higashiguchi, H. Kayano and T. Onchi, J. Nucl. Mater., 80, 24 (1979).

9. F. Onimus, I. Monnet, J.L. Be'chade, et al., J. Nucl. Mater., 328, 165 (2004).

10. D. Hull and D.J. Bacon, Introduction to Dislocations, $4^{\text {th }}$ edition, ButterworthHeinemann, Oxford (2001).

11. G.J. Ackland, S.J. Wooding and D.J. Bacon, Phil. Mag. A, 71, 553 (1995).

12. Yu.N.Osetsky and D.J. Bacon,. Modelling Simulation Mater. Sci. Eng., 11, 427 (2003).

13. M. I. Mendelev and G. J. Ackland, Phil. Mag., 87, 349 (2007).

14. H. Khater, Ph.D. Thesis, The University of Liverpool, 2008.

15. A. Serra and D.J. Bacon, Acta Metal. Mater., 43, 4465 (1995).

16. A. Serra, D.J. Bacon and R.C. Pond, Acta Mater., 47, 1425 (1999).

17. N. de Diego, Yu. N. Osetsky y D.J. Bacon, J. Nucl. Mater., 374, 87 (2008). 


\section{Figure captions}

Fig. 1. $[\mathbf{1 2} 10]$ projection of the dichromatic pattern formed by two black and white interpenetrating HCP lattices with a common (10,12) plane. The outline of two HCP unit cells is shown. Vectors $\boldsymbol{b}_{1 / 0}$ and $\boldsymbol{b}_{-1 / 0}$ are two of the $1 / 3<\mathbf{1 1 2 0}>$ Burgers vectors of perfect dislocations in the white crystal: $\boldsymbol{b}_{-7 /-8}, \boldsymbol{b}_{-5 /-6}$ and $\boldsymbol{b}_{-2 /-2}$ are Burgers vectors of interfacial disconnections formed by bringing together the steps shown on the lower surface of the white crystal and upper surface of the black crystal. A twinning dislocation has $\boldsymbol{b}=$ either $\boldsymbol{b}_{-2 /-2}$ or $\boldsymbol{b}_{+2 /+2}$.

Fig. 2. Schematic illustration of the reaction products found in [16] when an infinitelylong, $60^{\circ}$-mixed crystal dislocation interacts with a $(10 \overline{1} 2)$ twin boundary.

Fig. 3. Near-x-axis visualisation of the twin boundary when first a $60^{\circ}$-mixed $\boldsymbol{b}_{-1 / 0}$ crystal dislocation is absorbed to form a $\left(\boldsymbol{b}_{4 / 4}+\boldsymbol{b}^{\mathrm{L}}\right)$ complex and then a second dislocation of opposite sign $\left(\boldsymbol{b}_{1 / 0}\right)$ is absorbed to form a $\boldsymbol{b}_{-5 /-6}$ disconnection. The trailing $30^{\circ}$ partial of the first dislocation is seen to be standing away from the interface. The nature of the defects involved is indicated by the usual dislocation symbols. Atoms in the interface are visualised and coloured by their coordination number.

Fig. 4. (a, b) Visualisations of interface and dislocation atoms showing two stages when a twin boundary meets and passes through an interstitial dislocation loop with $\boldsymbol{b}$ parallel to the x-axis. (c) The configuration in (b) in z-axis projection: the curved shape of the twinning dislocation gliding from left to right demonstrates the obstacle strength the loop provides to twinning. (d) Alternative to (a) when the order of the Shockley partials making up the basal-plane sides of the loop is reversed. The Shockley partials are seen in (c) and (d).

Fig. 5. (a,b) Visualisation of interface and dislocation atoms showing two stages in the interaction between a twin boundary and a vacancy dislocation loop with $\boldsymbol{b}$ parallel to the x-axis. The loop is seen to be smaller in (b) after the interaction due to the detachment of vacancies on the left and right. (c) Change in potential energy of the 
model with applied shear strain during boundary interaction with interstitial and vacancy loops.

Fig. 6. Visualisations of the interaction between the twin boundary and interstitial loops with $\boldsymbol{b}$ at $60^{\circ}$ to the $\mathrm{x}$ axis. (a) The loop in the model with the M07 interatomic potential is pushed on its glide prism ahead of the boundary. In the model with the A95 potential, either (b) one side of the loop is trapped in the boundary or (c) all sides are absorbed, depending on the order of the basal-plane Shockley partials. In both cases, the interstitials are transported along the glide prism of the original loop as the interface moves up or down.

Fig. 7. (a) Near-X-axis visualisation after absorption of a vacancy loop with $\boldsymbol{b}$ at $60^{\circ}$ to the $\mathrm{x}$ axis in the twin boundary. A $\boldsymbol{b}_{-2 /-2}$ twinning dislocation is approaching from the left. (b) $\mathrm{z}$-axis visualisation showing the asymmetric shape of the $\boldsymbol{b}_{-2 /-2}$ twinning dislocation as it passes through the periodic row of loops. Two model cells are set sideby-side to make the dislocation shape clearer. 
FIGURE 1

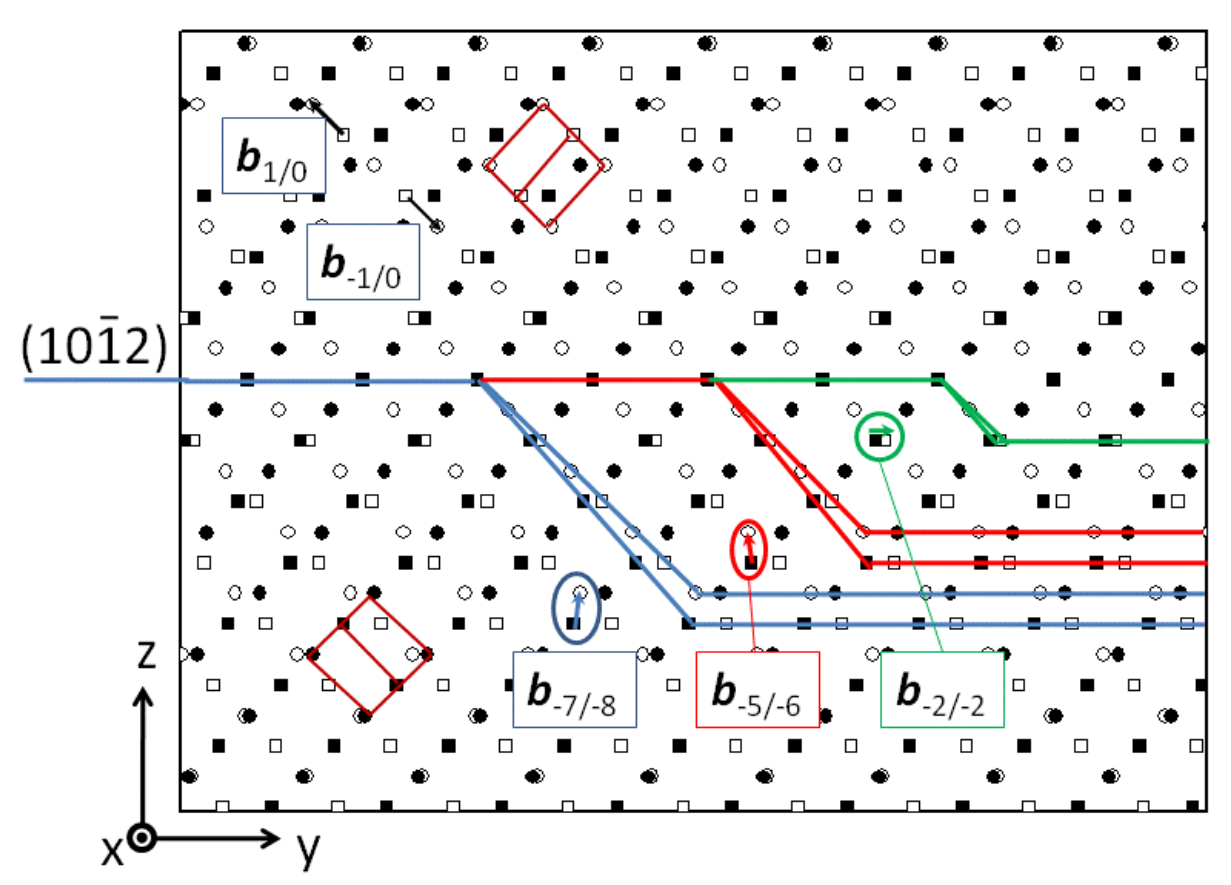




\section{FIGURE 2}
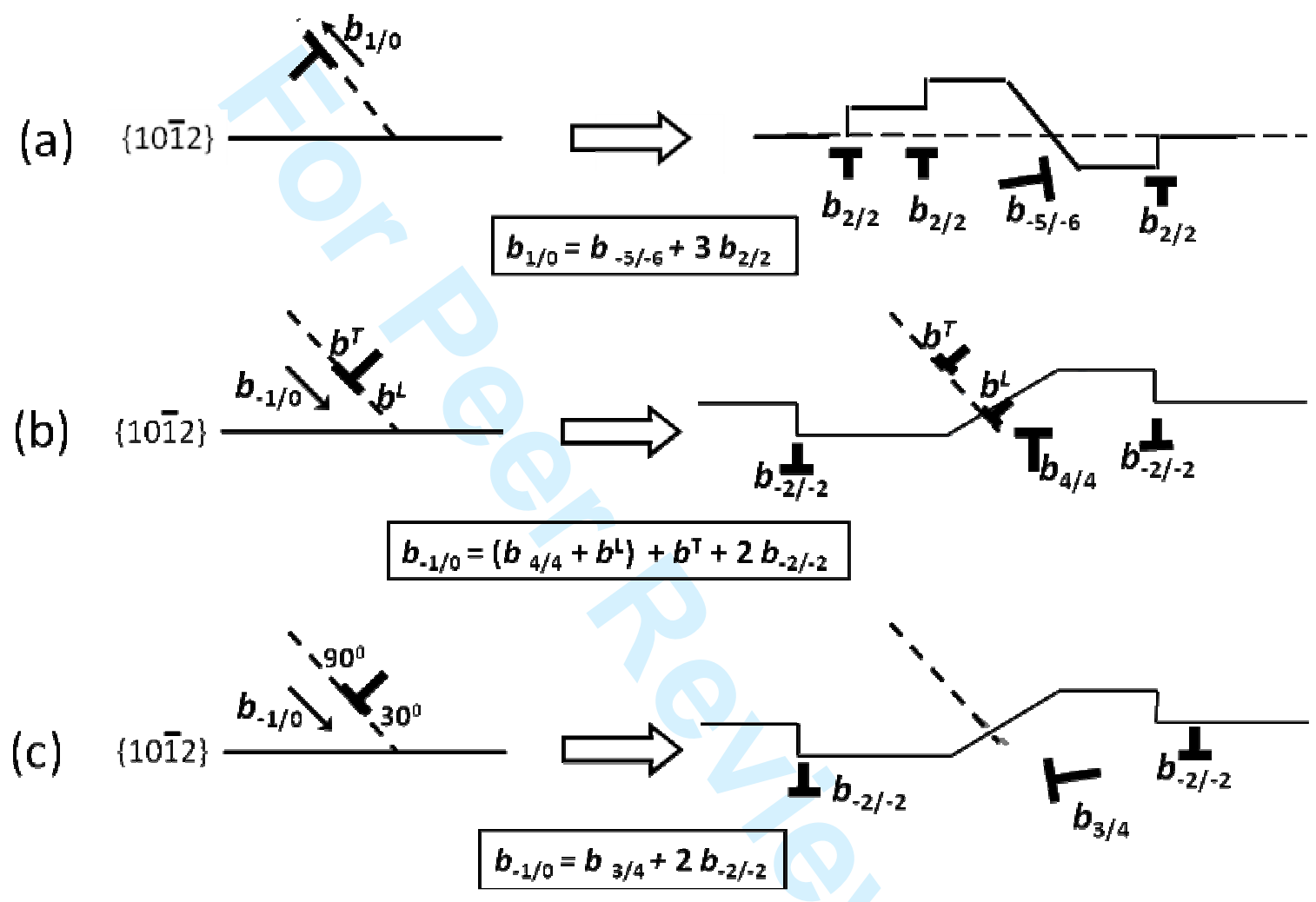
FIGURE 3

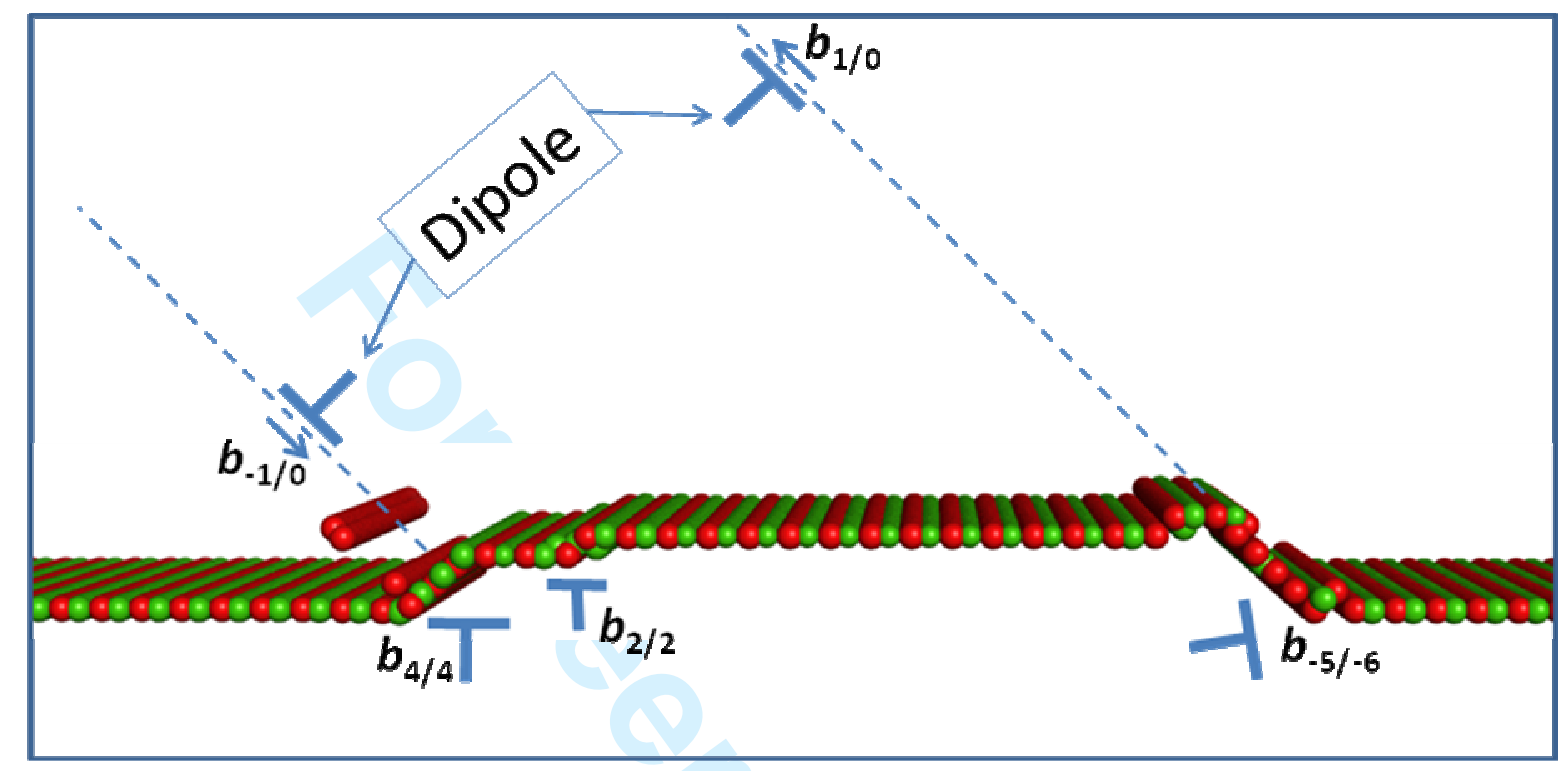




\section{FIGURE 4}

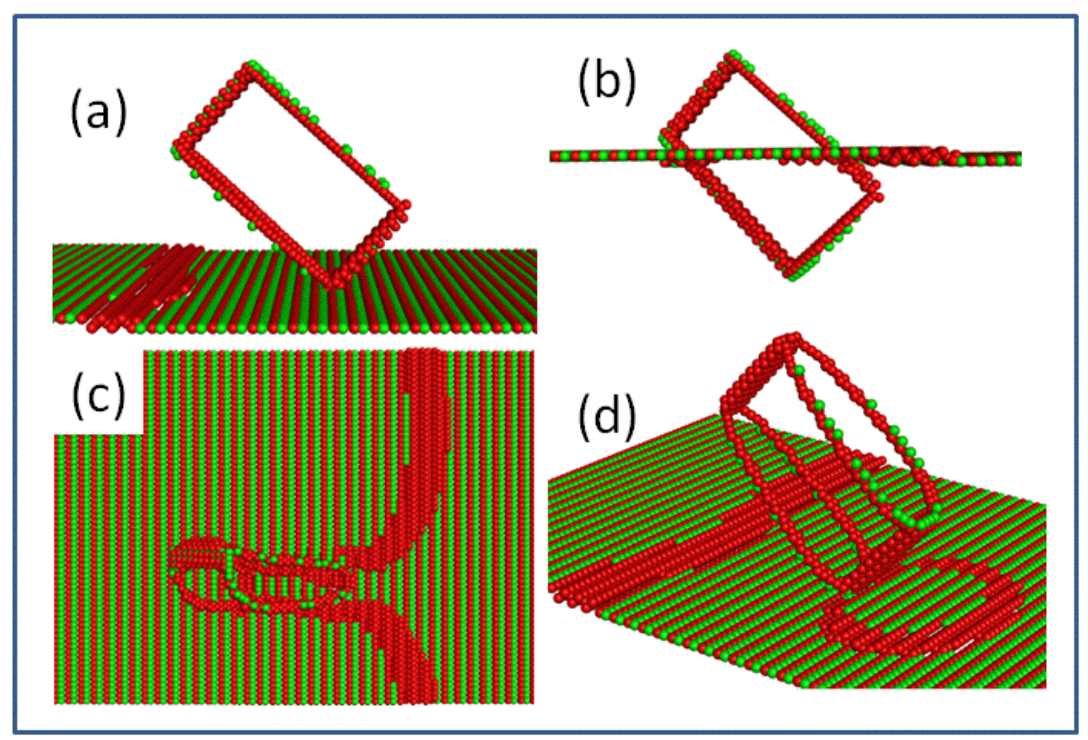


FIGURE 5
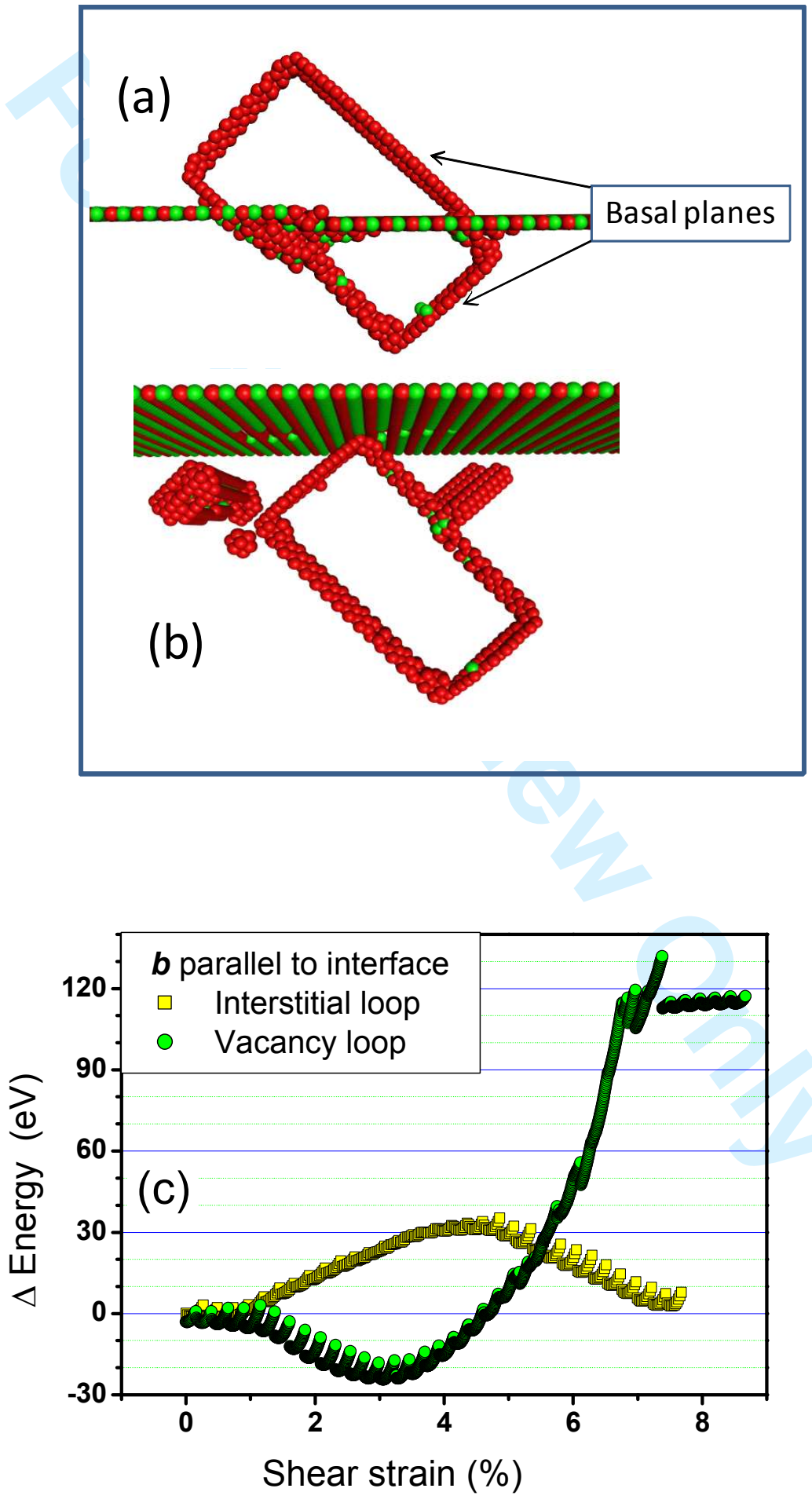


\section{FIGURE 6}

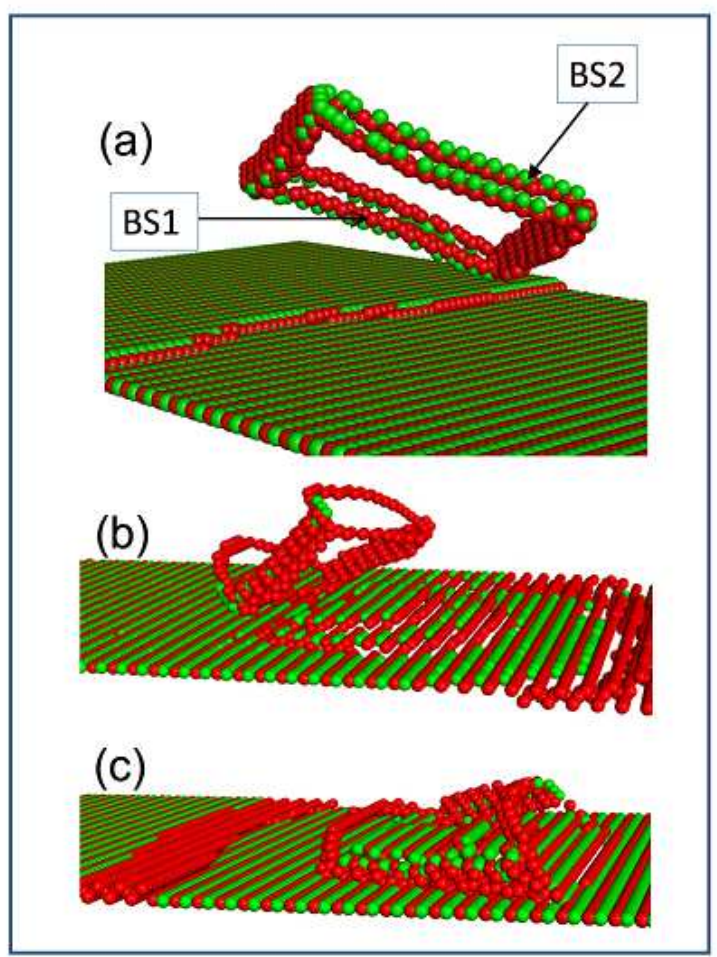




\section{FIGURE 7}

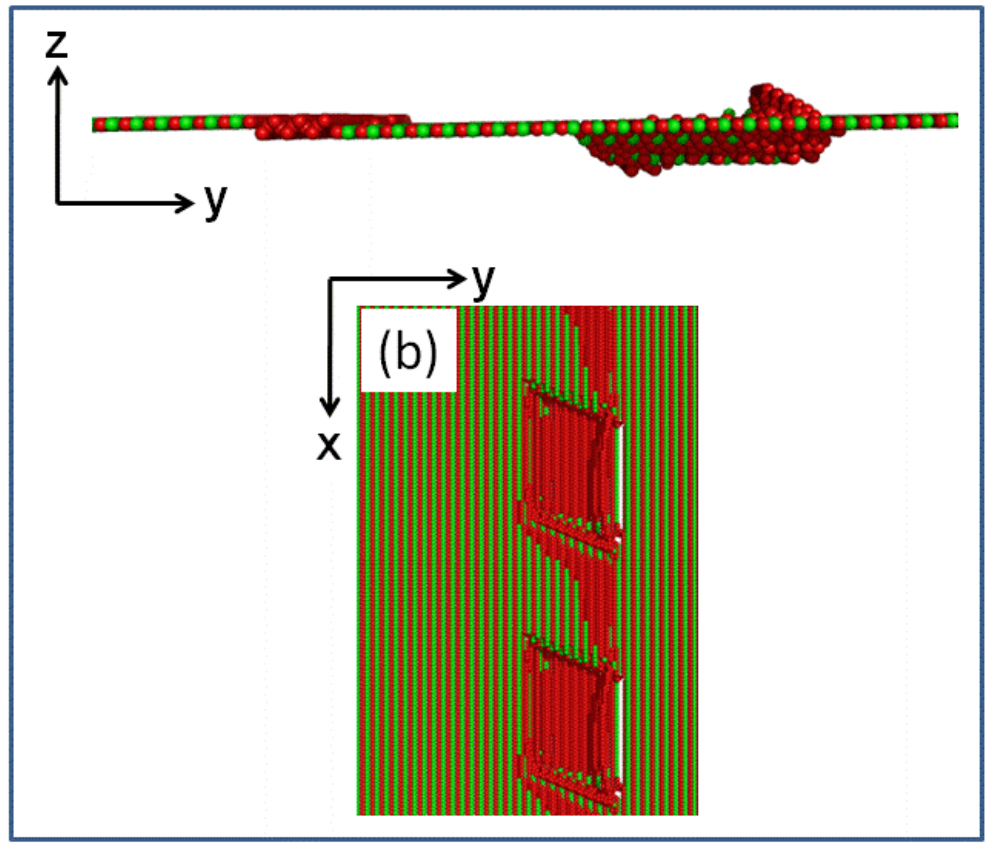

